\title{
LA PRESENCIA DE LOS MIGRANTES LATINOAMERICANOS EN GÉNOVA-ITALIA: EL CASO DE PERÚ Y ECUADOR
}

\section{IVONNE VALENCIA LEÓN}

Universidad Nacional Mayor de San Marcos. Lima (Perú).

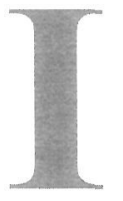

\section{RESUMEN}

talia es uno de los países europeos de mayor atracción migratoria, siendo el colectivo peruano el más numeroso de los países latinoamericanos y el colectivo ecuatoriano el más numeroso en cuanto al crecimiento de población en los últimos 5 años. Italia ofrece grandes oportunidades de desarrollo, especialmente para los migrantes jóvenes, quienes son protagonistas de una etapa muy particular en las sociedades globalizadas. Como resultado del proceso migratorio, las familias peruanas y ecuatorianas se han adaptado a las ciudades de destino y se han producido cambios en el interior de su organización, como el rol de la madre, la desintegración familiar y la nueva constitución de familia (mixta). La población migrante es básicamente joven y femenina y busca alcanzar el sueño europeo.

Palabras clave: ecuatorianos y peruanos en Europa, Italia, Génova, procesos de inclusión.

\section{LA MIGRACIÓN INTERNACIONAL}

La migración internacional de latinoamericanos es un fenómeno que se incrementó rápidamente desde los años 60 y 70 . En esa época, el $67 \%$ de los migrantes se establecían en los países fronterizos como Venezuela y Argentina. Incluso, los gobiernos impulsaron una política de estímulo a la migración de mano de obra cualificada. A partir de los años 80, el flujo de migrantes se orientó hacia Estados Unidos, Canadá, Europa, Australia y Japón. En los años 90, la emigración hacia los países desarrollados se agudizó, siendo Estados Unidos el de mayor preferencia. Así, los latinoamericanos registrados en Estados Unidos en 1960 eran un millón, mientras que en 1990 llegaron a ser ocho millones y medio (Pellegrino, 2002-3.2.9). 
Según el Censo de Italia de 2001, sobre la estructura demográfica y familiar de la población extranjera residente ${ }^{1}$, existen 1.334 .889 ciudadanos extranjeros residentes en Italia. El 35,1\% de esta población reside en el nor-oeste (Milán, Torino y Brescia). Del total, 586 mil personas son europeas y más de 122 mil residentes son latinoamericanos (peruanos, brasileños, ecuatorianos y dominicanos). Según esta misma fuente, Perú se encuentra entre las primeras diez comunidades extranjeras. Maruecos, Albania, Rumania, Filipinas, Yugoslavia, Túnez, China, Alemania, Senegal y Perú son los colectivos migrantes de mayor presencia en Europa. Entre los motivos por los que han migrado a Italia se encuentran: trabajo $(46.6 \%)$, reagrupación familiar $(36.5 \%)$, estudio $(3.1 \%)$ y otros motivos (religioso y asilo político) (13.8\%). El 97.9\% del total de extranjeros en Italia vive con su familia. En la región de la Lombardía, se encuentran 104 mil familias cuyos componentes son todos extranjeros. Sin embargo, el $45.1 \%$ del total de las familias italianas cuentan por lo menos con un miembro de nacionalidad extranjera debido a los matrimonios mixtos. La población migrante es básicamente joven. Las edades de los migrantes fluctúan alrededor de los 30 años (30,4 para varones y el 31,4 para mujeres), lo que indica que la mayor parte de los migrantes son adultos jóvenes.

\section{LA MIGRACIÓN PERUANA A ITALIA}

En los primeros años de migración, los peruanos que dejaron el país eran profesionales y técnicos de clase media ${ }^{2}$, pero los que siguen llegando a Italia son de las zonas populares, urbano-marginales y de Los Andes; todos ellos en busca de mejoras económicas con la esperanza de alcanzar el bienestar que el país no les pudo ofrecer. La mayoría se presentan al Consulado General de Italia en Lima con un contrato de trabajo o con una invitación para hacer turismo. Ya en Italia, se encuentran frente a otra dinámica social, deciden arriesgar todo y pasar a la clandestinidad. Por ejemplo, algunos integrantes de grupos de danzas folklóricas que fueron contratados por una corta temporada decidieron quedarse. Una vez que se termina el plazo de la visa, y de no haber obtenido el permiso de estadía, su situación irregular les impide conseguir un trabajo debidamente remunerado. El desconocimiento del idioma es un factor que limita el proceso de asimilación en Italia. Aprender el italiano es un reto, una vez que lo logran atraviesan la frontera más difícil.

I Instituto Nazionale di Stadistica, La presenza straniera in Italia: caratteristiche socio-demografiche. Roma, 2004.

2 César Germaná (2004) realizó un estudio sobre la migración internacional cualificada de peruanos. En este trabajo, intenta cuantificar la mano de obra cualificada (profesionales y técnicos) que reside fuera del Perú. 
Es a partir de ese momento cuandocomienza su propia asimilación. A pesar de las dificultades que encontraron, el sueño europeo los anima y los motiva a seguir adelante.

Muchos de los peruanos que residen fuera del país tienen una experiencia migratoria anterior porque se trasladaron del campo a la ciudad. Por lo general, también han migrado a países fronterizos como Chile, Venezuela, Bolivia, Paraguay y Brasil. Después de este proceso, decidieron migrar a Italia. Esta población es originaria de los principales departamentos: Cusco, Arequipa, Lima y Trujillo.

Debemos diferenciar dos tipos de migrantes peruanos. De una parte, los que viajaron hacia Estados Unidos, que en su mayoría son varones. Por otro lado, los que optaron por establecerse en Europa, donde las opciones laborales están dadas básicamente para la mano de obra femenina. El colectivo peruano es el de mayor número de migrantes latinoamericanos. En la década pasada, en Génova se encontraba la comunidad peruana más numerosa. Actualmente sólo residen alrededor de 4.000 migrantes. Estas cantidades son aproximadas, en la medida en que difieren del porcentaje de registrados en los Consulados de Perú en Italia.

\section{LA MIGRACIÓN ECUATORIANA A ITALIA}

El fenómeno migratorio de Ecuador tuvo sus inicios en 1950. En ese entonces, los pobladores de Azuay, fabricantes de sombrero de paja, tuvieron que enfrentar una crisis de comercialización, siendo los productores los primeros en abandonar el país con dirección a Estados Unidos. El Acuerdo Hispano-Ecuatoriano de 1963 permitió que muchos ecuatorianos viajaran a España sin visa por un período de 90 días. Este tipo de "ventajas" animó a muchos a quedarse, a costa de pasar a la clandestinidad. Más tarde, la aguda crisis socioeconómica del país motivó la partida de personas calificadas y no calificadas para trabajar en países más desarrollados.

A fines de siglo XX, Ecuador enfrenta la peor crisis de su historia. Según la Cartilla sobre Migración $\mathrm{N}^{0} 3$, sobre las causas del reciente proceso emigratorio ecuatoriano (2003-2) ${ }^{3}$, en 1982, Ecuador vivió un período de estancamiento y fue en 1999 cuando registra la mayor caída del PBI. La población ecuatoriana atravesó por una etapa de empobrecimiento, que en el ámbito latinoamericano fue el más acelerado de su historia. Es en este contexto que casi dos millones y medio de ecuatorianos abandonaron su país. Según la Cartilla sobre Migración $\mathrm{N}^{\mathrm{0}} 7$, sobre

3 ILDIS-FES Y SJM. Han publicado siete Cartillas sobre Migración, donde se analizan las características de la migración ecuatoriana en los diferentes paises de destino y los efectos socioeconómicos en Ecuador. 
el proceso migratorio en el sur de Quito (2004), al menos el $10 \%$ de la población ecuatoriana ha emigrado. Un importante grupo se dirigió hacia los Estados Unidos. Posteriormente, optaron por establecerse en países europeos, siendo España el que concentra la mayor cantidad de población ecuatoriana migrante. Italia es otra de las sedes de mayor preferencia para este colectivo. Actualmente, residen en Italia aproximadamente 60.000 ecuatorianos. Hay quienes señalan que esta cifra se duplica debido a los ciudadanos en condición irregular. Según información del Consulado General de Ecuador en Génova, existen 20.000 ecuatorianos residentes en esta ciudad, siendo esta comunidad extranjera la más numerosa en Génova.

El Censo de Italia de 2001, sobre la estructura demográfica y familiar de la población extranjera residente ${ }^{4}$, señala que 12.281 ciudadanos ecuatorianos obtuvieron el permesso di soggiorno en enero del año 2003. Esto significa que la condición de una parte de los ecuatorianos es irregular.

Las ocupaciones laborales de la población latinoamericana migrante están predeterminadas: actividades domésticas y construcción civil. Estos trabajos necesitan mano de obra no cualificada. Aunque las condiciones laborales para muchos no son las mejores, les permiten enviar dinero a sus familias en Ecuador. Así, la segunda fuente de divisas, luego del petróleo, son las remesas de los migrantes en el exterior. De esta manera, las remesas constituyen un ingreso familiar y nacional de vital importancia.

Una de las motivaciones para migrar del Perú y de Ecuador son las mejores oportunidades de educación para los hijos. La educación técnica es una apuesta de casi todos los migrantes. Los estudios en algunas escuelas públicas ofrecen la oportunidad de concluirlos con un diploma de técnico en electricidad, en mecánica, en turismo, entre otras especialidades.

Pablo dijo "en el Perú uno estudia, pero, cuando termina, no consigue trabajo. Aqui el trabajo es seguro, por eso el diploma vale más..."

La imagen que tenían sobre la educación italiana fue sobrevalorada. Sin embargo, a partir de la experiencia, señalan que el prototipo de educación es muy diferente a la imagen tradicional que tenían en mente y que en realidad no es mejor. Todo lo contrario, es más flexible. Este enfoque no les ha gustado, pero consideran que estudiar fuera siempre es mejor, ya que el diploma "vale más". Este valor simbólico que le dan al diploma escolar está en función de conseguir un empleo de manera

4 Instituto Nazionale di Stadistica, La presenza straniera in Italia: caratteristiche socio-demografiche. Roma, Giugnio 2004 
inmediata al egreso. Seguir estudios universitarios es sólo una ilusión. Muchos quisieran estudiar pero no pueden porque tienen que trabajar para ayudar a su mamá a mantener a la familia.

En suma, las crisis socioeconómicas de Perú y Ecuador motivaron la partida de personas cualificadas y no cualificadas, las cuales se encuentran realizando actividades laborales (la mayoría de ellos) con el ánimo de alcanzar el bienestar familiar en torno a un proceso de inserción en las sociedades de destino. En el cuadro 1, podemos apreciar la población de América Centro Meridional que obtuvo permesso di soggiorno hasta el 1 de enero de 2003. Se observa que la población que solicitó el permiso para trabajar en Italia es mayoritariamente femenina y peruana, que equivale al $2 \%$ de la población migrante en Italia. Esto se debe a la demanda de la mano de obra para realizar las labores domésticas y el cuidado de niños y ancianos. El colectivo femenino de Ecuador es cuantitativamente superior a la población masculina, lo que indica que el mayor porcentaje de mano de obra ecuatoriana en Italia es femenino.

Cuadro 1. Permesso di soggiorno por género al 1 de enero de 2003

\begin{tabular}{l|c|c|c} 
PAÍS DE & $\begin{array}{c}\text { POBLACIÓN } \\
\text { PASCULINA }\end{array}$ & $\begin{array}{c}\text { POBLACIÓN } \\
\text { FEMENINA }\end{array}$ & $\begin{array}{c}\text { TOTAL POBLACIÓN } \\
\text { MIGRANTE }\end{array}$ \\
\hline Argentina & 5.292 & 5.974 & 11.266 \\
Brasil & 5.428 & 15.513 & 20.941 \\
Colombia & 3.303 & 8.187 & 11.490 \\
República Dominicana & 2.761 & 9.033 & 11.794 \\
Ecuador & 3.753 & 8.528 & 12.281 \\
Perú & 10.627 & 20.696 & 31.323 \\
América Centro Meridional & 39.651 & 89.712 & 129.363 \\
\hline
\end{tabular}

Elaboración propia. Datos del Instituto Nacional de Estadística ${ }^{5}$

Según Caritas $^{6}$, la presencia latinoamericana entre 1993 y 2003 cuantitativamente es la más relevante en Europa. Podemos observar en el cuadro comparativo 2 que, a partir del año 1998, el colectivo peruano es el más numeroso de la población latinoamericana en Italia. La comunidad ecuatoriana, el incremento mayor se ha producido en el año 2003 con 45.859 migrantes residentes en Italia. Podemos observar también el significativo incremento de la población migrante en todos los casos en el año 2003. Según el mismo informe, la población femenina constituye el $48 \%$ del total de la comunidad latinoamericana en Italia.

5 Reporte de permessi di soggiorno al 1 de genario 2003. ISTAT. Sobre los datos del Ministerio del Interior de Italia.

6 Caritas presenta en el Dossier Statistico 2004, cuyo slogan de presentación es: sociedad abierta, sociedad dinámica, información actualizada en torno a la población migrante en Europa. 
Cuadro 2. Presencia latinoamericana en Italia entre 1993 - 2003

\begin{tabular}{l|c|c|c} 
PAís DE AMÉRICA DEL SUR & $\begin{array}{c}\text { ITALIA } \\
\mathbf{3 1 - 1 2 - 1 9 9 3}\end{array}$ & $\begin{array}{c}\text { ITALIA } \\
\mathbf{3 1 - 1 2 - 1 9 9 8}\end{array}$ & $\begin{array}{c}\text { ITALIA } \\
\mathbf{3 1 - 1 2 - 2 0 0 3}\end{array}$ \\
Argentina & 6,576 & 5,725 & 14,553 \\
Brasil & 11,495 & 16,593 & 26,858 \\
Colombia & 5,062 & 7,522 & 15,182 \\
Ecuador & 1,280 & 4,908 & 45,859 \\
Perú & 6,163 & 23,637 & 46,964 \\
Venezuela & 2,189 & 2,654 & 4,462 \\
Otros países & 2,464 & 2,701 & 8,802 \\
Total & $\mathbf{3 5 , 2 2 9}$ & $\mathbf{6 3 , 7 4 0}$ & $\mathbf{1 6 2 , 2 8 0}$
\end{tabular}

Elaboración propia. Datos del Dossier Statistico 2004 de Caritas.

Si bien, cuantitativamente, los colectivos peruano y ecuatoriano en Italia son disímiles, las motivaciones para dejar el país de origen y enfrentar nuevos retos son muy parecidas. Al ser países fronterizos y geográficamente similares, con los mismos problemas socieconómicos, con las mismas características y con dos millones y medio de migrantes fuera de cada país, nos permite establecer algunos rasgos comunes. Sin embargo, los tiempos en que esta masiva población se desplaza hacia Europa y específicamente hacia Italia son diversos. Los años de experiencia migratoria son distantes entre sí. En tanto, las generaciones determinan la tipología de los migrantes. Los que se fueron hace veinte años atrás encontraron otra sociedad; los que llegaron hace cinco años y los que siguen llegando tienen que enfrentar a una sociedad que ya no tiene la misma predisposición de años anteriores, ni tampoco les brinda la acogida que esperaban.

\section{LA FAMILIA PERUANA Y ECUATORIANA MIGRANTE EN GÉNOVA}

La mayoría de los migrantes que residen en la ciudad de Génova viven con casi toda su familia. En algunos casos comparten la casa con tíos y primos. Por lo general, habitan viviendas de mediana dimensión que comparten hasta con 10 personas, especialmente durante el primer año de estancia. La madre vivía con amigas o algún familiar, pero al llegar los hijos se mudaron a otra casa, con otras personas. La idea de compartir la vivienda es para poder compartir también el costo del alquiler. Cuando los padres encontraron un trabajo estable, pudieron alquilar una casa. La familia migrante, antes de establecerse en algún lugar definitivo, ha tenido hasta cinco cambios de domicilio.

Sobre las entrevistas realizadas, un importante porcentaje de familias migrantes están destruidas, fragmentadas o en proceso de constitución. En general, las familias peruanas están más unidas que otras familias 
latinoamericanas. En el caso ecuatoriano, encontramos que el $80 \%$ de familias de los adolescentes entrevistados están fragmentadas. El $60 \%$ de ellas estaban destruidas antes de migrar. La mayoría de las madres son separadas y otras se han vuelto a casar. El nuevo compromiso no necesariamente es italiano sino de la misma nacionalidad. En el caso peruano, encontramos que la mayoría de las familias se han mantenido unidas a pesar de las dificultades. Algunas personas señalaron que los problemas fortalecieron la relación. Si bien el nivel de divorcios y sobretodo el de separaciones el muy alto en el Perú, la familia migrante en Génova se estableció hace 10 y 15 años, tiempo en el que las relaciones se consolidaron o se han vuelto a constituir y ahora son familias estables. Sin embargo, los matrimonios jóvenes o las familias que tienen 5 años o menos de residencia son las se encuentran desarticuladas. Por otro lado, muchas familias son italo-peruanas, ya que se han constituido matrimonios mixtos. A finales del año $2003^{7}, 382$ peruanos han adquirido la ciudadanía italiana. De ellos, 350 la obtuvieron por haber contraído matrimonio con un italiano(a). Este tipo de familias se puede apreciar en las actividades peruanas. Por ejemplo, en la procesión de Señor de los Milagros, se observa la participación de la comunidad italiana debido a las mixturas sociales.

Creemos que la migración no siempre es causa del rompimiento familiar. Para las familias que estaban anteriormente destruidas, la migración ha sido una salida de emergencia a todos sus problemas, con la finalidad de empezar una nueva vida, llena de ilusiones. En general la familia está muy dañada, son pocos los matrimonios que están unidos, sin embargo, en Génova residen familias peruanas completas que han alcanzado un nivel de vida adecuado. Por lo tanto, atribuimos la estabilidad de la familia peruana migrante a la cantidad de años de residencia en Génova.

El concepto de familia nuclear en términos migratorios sufre una alteración, en la medida en que casi no existe. La necesidad por cubrir los gastos motiva la convivencia de otros miembros de la familia como los primos, sobrinos, tíos, cuñados que se encuentran en proceso de adaptación. En este sentido, el tipo de familia extensa es propio para definir el perfil de la familia migrante, aunque existe una tendencia a la constitución de nuevas familias nucleares al separarse los hijos mayores de los padres. El factor de solidaridad y ayuda mutua posibilita el tipo de familia extensa. La solidaridad entre hermanas que tienen hijos es una característica notoria entre las peruanas. En este caso, los abuelos no participan de esta nueva familia-digamos-italo-peruana porque han

7 CARITAS/MIGRANTE 2004. Dossier Statistico Inmigrazione 2004. Elaborazione su dati del Ministerio de'll Interno, 2004. 
sido el soporte que les permitió a las madres dejar el país, al encargarse de los hijos, especialmente de los más pequeños. En algunos casos, sólo han podido visitarlos, pero no podrían residir en Italia por la sencilla razón de que no tienen quien los cuide y porque se convertirían en una carga familiar.

La población europea se caracteriza por ser longeva. Es por eso la importancia de la mano de obra, especialmente femenina, que se encarga de sus cuidados. Actualmente, el $65 \%$ de los migrantes peruanos en Italia es del sexo femenino ${ }^{8}$. La población migrante es mayoritariamente joven y tiene que adaptarse a una sociedad que envejece. La edad promedio de la población italiana es superior a la migrante. Para el ISTAT $^{9}$, la edad media asciende a 42 años, $(40,1$ para los varones y 43,1 para las mujeres). Basta viajar en el ómnibus para ver que la mayor parte de pasajeros son ancianos. La otra parte la constituyen los jóvenes migrantes. Las Naciones Unidas señala que el $37 \%$ de la población española tendrá más de 65 años en el año 2050. España será uno de los países más envejecidos del mundo, junto con Japón (36.4\%) y con Italia $(35.9 \%)^{10}$.

Sobre las entrevistas realizadas, algunas instituciones que se ocupan de la oferta y la demanda de trabajo doméstico aseguran que la mujer peruana es muy cotizada en el momento de selección de personal, ya que son las más solicitadas por las familias italianas. Las maestras o las que tienen algunos estudios superiores son requeridas para cuidar niños. Muchas de estas mujeres son madres, por lo que tienen experiencia y disposición para tratar adecuadamente a los niños. Lo mismo sucede con la selección para el cuidado de ancianos.

Los peruanos y ecuatorianos que han logrado una estabilidad familiar y económica regresan al país para reencontrarse con sus familias y amigos, pero sólo de vacaciones. Este breve retorno les da un sentido de distinción y de reconocimiento superior al que tenían antes de la partida. El volver a casa después de "haber conquistado el mundo" los hace sentir diferentes. El -digamos- permanente intercambio lo convierte en un agente que transporta cultura y estilos de vida acá y allá. Estas personas han matizado las ciudades donde habitan y a la vez llevan consigo conocimientos y costumbres adquiridas en Italia. Los matrimonios trasnacionales son los principales agentes de este proceso. La posibilidad de retorno definitivo es algo que la mayoría no considera. Al contrario,

8 Actualmente los migrantes peruanos presentes en ltalia son 49.964 , de ellos el $70.3 \%$ son mujeres (CaritasMigrantes 2004).

9 Instituto Nazionale di Stadistica, La presenza straniera in Italia: caratteristiche socio-demografiche. Roma, Giugnio 2004.

10 SÁNCHEZ, Mayka, "Hacia una población cada vez más envejecida". Entorno Social. España. 2003. 
lo mejor para ellos es que toda la familia extensa pueda migrar. Incluso hay quienes quieren borrar su historia pasada y han adoptado, además de la nacionalidad italiana, toda su idiosincrasia.

Los latinoamericanos no sólo migraron para trabajar, también migraron con sus costumbres, su cultura y su estilo de vida. Han transportado su folklore, tradición, ideología y asumieron algunas otras propias de la ciudad de destino, lo que en general no les da autenticidad, pero que, poco a poco, van forjando su propia identidad, de acuerdo a las circunstancias por las cuales atraviesan y en los espacios en los que se desarrollan.

\section{EL ROL DE LA MADRE EN LA FAMILIA MIGRANTE}

La mujer, especialmente la madre, es la que apuesta por una nueva vida. Por lo general, es motivada por algún familiar o amistad que se encontraba trabajando en Italia. Las madres se acogieron a la Ley de reagrupamiento familiar, motivadas por el cierto abandono de los hijos y la creciente violencia. Todos los migrantes legalmente inscritos tienen derecho a pedir a sus hijos menores de edad. En este sentido, los esposos y los hijos pueden reencontrarse con la madre. El traslado de los hijos es uno a uno por lo costoso de viajar a Europa. Es así que la madre se convierte en un elemento de atracción migratoria. Aunque, las condiciones laborales en Italia no les permiten estar juntos como ellos habían soñado.

Al salir la madre, los hijos se quedaron en el país bajo la tutela del padre, con alguna tía y en la mayoría de los casos con la abuelita. Muchos de los padres no vivían con ellos desde antes de la partida. La fragmentación familiar, en algunos casos, motivó la decisión de migrar. Los hijos mayores de edad viven en torno a la madre. Es importante destacar que la madre es el vínculo que los une a la tierra de origen. Si la madre se encuentra en el país de origen, entonces, los hijos siempre piensan en la posibilidad de retorno. Pero si la madre y toda la familia se encuentra en Italia, esa posibilidad casi no existe.

La familia migrante atraviesa por un cambio en su organización. La independencia femenina ha dado lugar a que los papás se encarguen del hogar por temporadas. Es decir, el rol de la madre en el interior de la familia se ha equiparado con el rol del padre, tradicionalmente establecido. Aunque en algunas ocasiones, este rol se ha invertido en el sentido que hemos encontrado a los papás a cargo de las cuestiones domésticas, del cuidado de los hijos, ayudarlos con los deberes escolares, el lavado de la ropa y la cocina. Esta situación se presenta cuando los 
padres no tienen trabajo; por tanto, es una cuestión más bien temporal. Mientras tanto, las mamás se encargan de llevar el dinero a la casa. Cuando ambos trabajan, comparten las actividades del hogar de manera igualitaria. Podemos señalar que la familia contemporánea es democrática. Sin embargo, cuando las familias son no estructuradas, la madre asume el doble rol. Esta situación trae consigo una serie de dificultades que tienen que enfrentar como puedan. Podemos decir que el rol de la madre en todos los casos es decisivo, ya que asume un rol autónomo e independiente. La idea tradicional de la mujer que se queda en casa al cuidado de los hijos ha pasado a la historia.

Debemos considerar que las familias originarias de los andes peruanos que migraron al exterior tienen un estilo de vida y de organización distinta a las familias de la costa. $\mathrm{Al}$ interior de estas familias impera un cierto machismo, que se resiste a ser dominado por la fuerza femenina. El jefe del hogar es siempre el padre, la figura materna no siempre es considerada en la toma de decisiones. En términos económicos, la mujer migrante tiene mayores ventajas que el hombre. Por lo que al interior de la familia tiene autonomía económica. Por eso decimos que la migración para el caso femenino es una liberación de la dominación masculina que las aguardó durante años. Por otro lado, esta situación afecta a los varones en la medida en que rompe los esquemas clásicos y los condiciona -en el mejor de los casos-a compartir con la mujer las tareas del hogar.

\section{CONSIDERACIONES FINALES}

La crisis económica y la violencia fueron los factores más importantes que motivaron la migración de peruanos al exterior. Dos millones y medio de peruanos viven fuera del país. Los profesionales y técnicos de clase media fueron los primeros en migrar. Sin embargo, los que llegaron en los últimos años y los que siguen migrando pertenecen a las zonas populares del país, especialmente de la región andina. Muchas de estas personas tienen una experiencia migratoria previa: migraron del campo a la ciudad, luego se trasladaron a algún país fronterizo y posteriormente se establecieron en Europa y Estados Unidos.

Italia se ha convertido en uno de los países de mayor atractivo migratorio para los latinoamericanos. La estabilidad económica, las opciones laborales, el nivel educativo para los hijos y la seguridad son algunos de los factores que motivan la migración de peruanos. La familia migrante se encuentra en un proceso de reorganización. La familia nuclear casi no existe, por tanto, el tipo de familia extensa es la mejor manera para 
definir el perfil de la familia migrante. En el nuevo contexto migratorio, la madre se convierte en un elemento de atracción migratorio y decisivo en el proceso de asimilación en la sociedad de destino. El rol de la madre migrante se ha transformado en autónomo e independiente. Por ello asume un papel preponderante al interior del contexto familiar. La migración peruana y ecuatoriana se caracteriza por una fuerte presencia femenina, la misma que se incrementa con el transcurrir del tiempo. En este sentido, podemos hablar de una feminización de la migración.

Las mujeres peruanas y ecuatorianas se dedican al cuidado de niños, de ancianos y de la familia italiana en general. Estas actividades se han convertido en el medio de vida de las migrantes. Muchas de estas personas son profesionales o tienen estudios técnicos, pero no pueden ejercer la carrera debido a que deben tramitar la reválida de su grado o seguir estudios de postgrado.

Una de las mejores motivaciones para quedarse en Italia es la educación de los hijos, la misma que está básicamente orientada a los estudios técnicos. El valor simbólico que otorgan a los diplomas es porque les da mayor seguridad para acceder a un trabajo inmediatamente después del egreso. Pero, muchos jóvenes, que constituyen esta segunda generación de migrantes, tienen problemas de incorporación en la sociedad. Debemos tener en cuenta que estos jóvenes son protagonistas de una época de transformación de la sociedad contemporánea y que el fenómeno de la migración los reta a adaptarse a una nueva sociedad.

La posibilidad de retorno definitivo al Perú es casi nula. En el mejor de los casos, el envío de remesas para sus familias es lo único que los mantiene relacionados con el país. La añoranza por los suyos los hace transportar sus costumbres y sus creencias, que se mezclan con el modo de vida italiano.

La comunidad italiana recibe a esta masa migratoria en un contexto particular. La movilización masiva de población hacia algunas ciudades está alterando su dinámica social. El colectivo peruano y el colectivo ecuatoriano considera a Italia como el país de grandes oportunidades, donde harán realidad el sueño europeo.

\section{BIBLIOGRAFIA}

ALTAMIRANO, T., 1996. Migración. El fenómeno del siglo. Peruanos en Europa. PUCP. Fondo Editorial. Lima.

ALTAMIRANO, T., 2000. Liderazgo y organizaciones de peruanos en el exterior. Culturas transnacionales e imaginarios sobre el desarrollo. PUCP. Fondo Editorial. Lima.

CARITAS/MIGRANTE, 2004. Dossier Statistico Inmigrazione 2004. Elaborazione su dati del 2004. Ministerio de'll Interno. 
CARTLLAS SOBRE MIGRACIÓN. Plan Migración, Comunicación y Desarrollo. ILDES-FES, Ecuador.

2002 El trabajo doméstico en la migración

2002 Las remesas de los emigrantes y sus efectos en la economía Ecuatoriana

2003 Causas del reciente proceso migratorio ecuatoriano

2004 El proceso emigratorio en el sur de Quito

GERMANÁ CAVERO, C., 2004. "Una aproximación a la migración internacional calificada en el Perú". En Studi Emigrazione. Centro Studi Emigracione, Roma 2004.

INSTITUTO NAZIONALE DI STADISTICA, 2004 La presenza straniera in Italia: caratteristiche socio-demografiche, Roma.

MEDI, Centro Studi Migrazioni nel Mediterráneo, 2003. "L' inmigrazione latinoamericana in Italia. Spunti di analisi statistico-demografica". Di Alessio D'Angelo.

SÁNCHEZ, M., 2003. "Hacia una población cada vez más envejecida". Entorno Social. España.

PELLEGRINO, A., 2002. "Drenaje, movilidad, circulación: nuevas modalidades de la migración calificada". Italia.

VALENCIA, 1., 2004. "Los adolescentes latinoamericanos en Génova". Ponencia presentada en el Convengo internazionale: I Latinos alla scoperta dell'Europa. Nuove migrazioni e spazi della cittadinanza., Italia.

VALENCIA, I.,2005. "Famiglie migranti peruviane in Italia: gli effetti positivi del tempo". En $l l$ fantasma delle bande. Gènova e i latinos. L. Queirolo y A. Torre. Fratelli, Frilli Editore, 2005, Génova, Italia. 